\title{
Estrés, Ansiedad e Insomnio: Asociación de enemigos ocultos que destruyen nuestro equilibrio mental. Reporte de caso.
}

\author{
Constanza Ballesteros Ospina, ${ }^{1}$ Rafael Lobelo García ${ }^{2}$ \\ Corresponding Author: Constanza Ballesteros Ospina, connieb72@gmail.com \\ ${ }^{1}$ CAYRE, Coordinadora de unidad de sueño pediátrica | connieb72@gmail.com \\ ${ }^{2}$ CAYRE, Director clinica de sueño | ralobelo@gmail.com
}

\begin{abstract}
Paciente de 46 años de edad que acude por cuadro de mas de 10 años de evolución con trastorno de sueño que consiste en dificultad para iniciar y de mantener el sueño. Esto asociado a trastorno de personalidad tipo ansioso. El caso clínico que traigo muestra la asociación entre estrés, ansiedad e insomnio, enemigos ocultos y cómo esta asociación deteriora la calidad de vida e impacta negativamente en el desempeño físico y mental de mi paciente y como con un manejo biorregulador podemos intervenir y cambiar favorablemente su condición de salud generando bienestar, recuperando su autoestima y nivelando su condición bio-psico-social. Durante el caso encontrarán el manejo farmacológico convencional que se le prescribió a la paciente por parte de sus especialistas y los efectos secundarios que estos generaron sin lograr control de la enfermedad y cómo la intervención con medicamentos biorreguladores con un equilibrio entre lo convencional y lo alopático lograron un éxito terapéutico sin efectos indeseables y recuperando su calidad de vida
\end{abstract}

Keywords: case report, stress, anxiety, insomnia, sleep disorder

\section{Introduction}

El insomnio es uno de los síntomas más prevalentes en la población general, estando entre un 25$70 \%$ de acuerdo al grupo poblacional evaluado. Es más frecuente en mujeres con una relación 3:1, y se exacerba durante la menopausia. En Colombia, se han documentado estudios donde la prevalencia general está en un 25\%, pero llega a ser del 50\% en poblaciones mayores de 50 años. El insomnio esporádico o situacional es muy frecuente y llega a estar presente en algún momento en la vida con una probabilidad del 70\%. El 6-9\% de los pacientes con síntomas de Insomnio, realmente presenta la enfermedad. La ansiedad puede tener una prevalencia entre el 25 al 44\%.La asociación entre estrés-ansiedad e insomnio tienen mayor riesgo de presentación con OR 2,97 a 4,60.

Los niveles de estrés se han documentado entre un 30 a 70\% de los pacientes evaluados, dependiendo de factores situacionales. La asociación entre insomnio y depresión es muy frecuente, de doble vía, con prevalencia hasta del $50 \%$, con mayor predominio en mujeres y personas mayores de 65 años. En la actualidad hay escasa evidencia de manejo del insomnio con medicina 
biorreguladora, hay series de casos descritas con fitoterapia y algunos productos homeopáticos, sin embargo, cada vez es mayor la búsqueda por parte de los pacientes de soluciones no tradicionales, especialmente por los efectos secundarios de los tratamientos.

\section{Timeline}

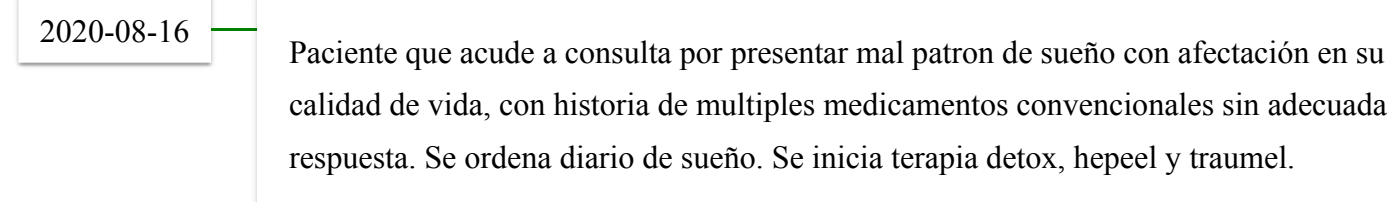

2020-08-30 - Se revisa diario de sueño y se mide escala de calidad de vida la que muestra deterioro importante en la escala mental y fisica. Se añade neurexan y nervoheel.

2020-09-21 - Acude a control refiriendo mejoría de la ansiedad con discreta mejoria en calidad de vida y aumento en numero de horas de sueño (4-5h). Refiere mejoria del $40 \%$ de sus síntomas iniciales. Se añade mirtazapina media tab en la noche.

2020-10-19 - Refiere franca mejoría en la calidad de sueño, en promedio 7 horas, desaparece el cuadro depresivo y de estres, considera que sus síntomas mejoraron en un $90 \%$. Se disminuye mirtazapina a $1 / 4$ de tableta.

2020-11-22 - Mantiene buena calidad de sueño, franca mejoría en la calidad de vida y mejoría en el diario de sueño y en la escala SF- 12. Refiere que sus sintomas mejoraron en mas del $98 \%$ de sus sintomas iniciales.

\section{$\underline{\text { Narrative }}$}

Se trata de una mujer de 46 años, natural de Cali, casada, con unión matrimonial estable, de profesión médica, Madre de dos hijos adolescntes y que labora en el área administrativa de una prestigiosa empresa de medicina prepagada.

Llega a nuestra consulta por insomnio. Refiere que tiene un cuadro de más de 10 años de evolución en donde presenta periodos intermitentes de cambios en la cantidad y calidad de sueño, en donde no logra dormir más de 3 horas. Los momentos de crisis pueden llegar a durar hasta 6 meses sin lograr mantener el sueño. Siempre ha estado asociado a su nivel de estrés laboral, ya que dirige los proyectos de expansión de una prestigiosa prepagada, en donde el nivel de exigencia es muy elevado. Ha sido valorada y tratada por psiquiatría y psicología, por lo que ha recibido múltiples 
tratamientos para el manejo de la ansiedad e insomnio con respuesta parcial y con efectos de letargia y somnolencia en el dia. Consulta por una nueva crisis en la que lleva 10 meses, con latencia de sueño de 2 horas, no mantiene el sueño, duerme 2 a 3 horas por noche, no tiene sueño reparador, en el día hay cansancio extremo, sin somnolencia diurna, con alteración en la concentración, cambios en su memoria, llanto frecuente, inestabilidad emocional y desde hace un mes ideas de desesperanza e incluso de no querer vivir, aunque no ha pensado en actos suicidas. Venía recibiendo manejo con sertralina, beozodiacepinas (clonazepam), levomepromacina, quetiapina, duloxetina, con lo que no mejoraba su condición de insomnio, permanecía con letargia lo que afectaba su desempeño laboral, le producía más depresión y estaba entrando en problemas en su relación matrimonial. No tiene antecedentes cardiovasculares, pulmonares ni metabólicos. No hay antecedentes de insomnio en su familia.Niega tabaquismo.Peso adecuado (IMC 23,4 mt2/kg). Es activa laboralmente, se considera una mujer exitosa, líder de grupo laboral, tene una personalidad ansiosa y esta sometida a alta carga de estrés laboral por logros de indicadores.

\section{Características del Sueño}

Se acuesta a las $10 \mathrm{pm}$, latencia de sueño de 1 a 2 horas, se despierta 2 a 3 veces por noche, duerme en promedio 2-3 horas, sueño no reparador, cefalea y embotamiento durante el día, no refiere pesadillas y no hay síntomas de MPP.

Higiene de Sueño

Se acuesta usualmente a la misma hora, ve TV hasta el momento que logra dormirse en la cama, suele trabajar en la noche "eso me cansa y me puede dar sueño", se da un baño en la noche lo cual la relaja, no toma bebidas estimulantes después de las $3 \mathrm{pm}$, buen hábito alimentario en la noche, suele ser desordenada a la hora de tomar el almuerzo, y realiza caminatas diarias en la mañana o en la tarde de 40 minutos. Evaluada por psquiatria durante varios años por trastorno de ansiedad y estrés crónico, depresión asociada al trastorno de insomnio secundario. Buen soporte y acompañamiento familiar. Está sometida a alto nivel de estrés laboral. Medicada con sertralina, clonazepam, duloxetina y le habían iniciado levomepromacina y posteriormente quetiapina.

La paciente en vista de la no mejoría decide suspender estos medicamentos e intentar con valeriana, pasiflora, té relajante. Suspende las consultas por psiquiatría por la sensación de somnolencia diurna con los medicamentos. Consulta por somnología después de haber suspendido la terapia farmacológica convencional. En nuestra evaluación encontramos un claro trastorno de estrés y ansiedad, depresión asociada al insomnio, higiene de sueño deficiente, ideas de minusvalía y de no querer vivir. Paciente con uso crónico de medicamentos citotóxicos y hepatotóxicos con taquifilaxia secundaria a la cronicidad y abuso. Impacto negativo en su calidad de vida en la esfera mental y física. Hay deterioro en su desempeño laboral y su esposo refiere un ambiente agresivo permanente asociado al trastorno de insomnio que viene deteriorando su relación, cambios con disminución de la líbido.

Se hace una evaluacion utilizando escalas de calidad de vida y medicion de somnolencia diurna excesiva y se solicitan laboratorios de inicio. Escala de somnolencia diurna de Epworth: 6/24 (normal) Escala de calidad de vida-Cuestionario de salud SF12: Salud Mental: 24\%, Salud Física:

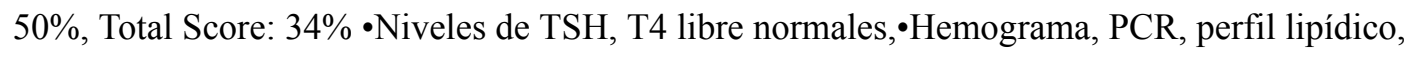


función renal, glicemia normales, Perfil hepático normal, Cortisol: 8 am: 24 mcg/dl, 5 pm: 18 $\mathrm{mcg} / \mathrm{dl}$. Niveles de vitamina D: $32 \mathrm{ng} / \mathrm{dl}$.

Enfoque terapeutico: Se interviene generando un ambiente de confianza, tranquilidad y paciencia. No se busca un efecto directo sobre el sueño. Se plantea Detoxificar y Biorregular en primera instancia. Se evitar el uso de medicamentos hipnóticos y benzodiacepinas e inmediatamente se trabaja en optimizar higiene de sueño.

El plan terapéutica instaurado fue el siguiente:

Detoxificación: Detox durante 8 semanas: Lymphomyosot, nuxel-homaccord, berberis-homaccord, Adicionamos Hepeel: tabletas 3 veces diarias, Neurexan: tabletas 3 veces diarias, Traumel: tabletas 3 veces diarias, esto durante 8 semanas Control a las 2 semanas: Refiere mantener el trastorno de sueño pero refiere franca mejoría en los niveles de ansiedad y de estrés, se mantiene terapia détox más hepeel, neurexan igual y nervoheel por asociación con depresión. Se realiaz nuevo control a las 4 semanas con mejoría notoria en la calidad de vida y ya duerme en promedio 4 horas. Desaparece la depresión, se mantiene la terapia instaurada y se inicia Mirtazapina 1/2 tableta en la noche.

Se realiza control a las 6 semanas: La paciente esta feliz, sin ansiedad ni depresión, duerme en promedio 7 horas, sueño reparador y en total control de su funcionamiento bio-psico-social. Se mantiene la terapia biorreguladora y bajo la dosis de mirtazapina a 1/4 de tableta En el control a las 8 semanas completa la terapia détox, se mantiene por 4 semanas la terapia con hepeel, mantengo neurexan y nervoheel. Duerme en promedio 7 horas y hay cambio total en su calidad de vida En la actualidad, repite terapia détox después de 8 semanas de haber terminado la primera terapia, se dio por 6 semanas junto con hepeel. Mantenemos neurexan con periodos de suspensión de un mes (3:1), ya se suepende el nervoheel y no toma mirtazapina. Mantiene 8 horas de sueño de muy buena calidad y esta totalmente activa sin trastorno depresivo ni de ansiedad.

\section{Diagnostics}




\begin{tabular}{|c|c|c|c|}
\hline 2020-08-08 & Eosinophils & 1 & $\%$ \\
\hline 2020-08-08 & Cholesterol, LDL & 89 & $\mathrm{mg} / \mathrm{dL}$ \\
\hline 2020-08-08 & Neutrophils & 66 & $\%$ \\
\hline $2020-08-08$ & T4, free (thyroxine) & 1.14 & $\mathrm{ng} / \mathrm{dL}$ \\
\hline $2020-08-08$ & Cholesterol, HDL & 68 & $\mathrm{mg} / \mathrm{dL}$ \\
\hline 2020-08-08 & $\mathrm{Hb}$ (hemoglobin) & 14.3 & $\mathrm{~g} / \mathrm{dL}$ \\
\hline 2020-08-08 & AST (aspartate aminotransferase) & 16 & $\mathrm{U} / \mathrm{L}$ \\
\hline $2020-08-08$ & Hct (hematocrit) & 43.9 & $\%$ \\
\hline
\end{tabular}

\section{Perspective}

Apreciados doctores: "Ha sido una gran fortuna su presencia en mi vida, hace 8 meses ( agosto del 2020) tenia una recaída en sueño, trastorno que padezco desde que termine mi año rural en 1.996 y que lo había manejado con inductores de sueño, medicamentos ansiolíticos e hipnóticos, pero coyunturalmente en el año pasado estaba en el peor de mis escenarios, solo dormía 3-4 horas, estaba totalmente deprimida y con deterioro en mi vida personal, sentimental y sexual. Con la medicación que usted me recetó y que juiciosamente cumplí y que he ido desescalonando, estoy durmiendo al rededor de 8 horas entre semana y el fin de semana descanso de corrido hasta las $8 \mathrm{am}$. Me levanto vital y recuperada, sin sensación de malestar ni cefalea. Mi vida personal y familiar han cambiado sustancialmente, tengo deseos de vivir y de trabajar y mi relación sentimental ha cambiado muy positivamente. Mi esposo los adora, y esta plenamente agradecido con ustedes. Como se los he dicho personal y telefónicamente, mil y mil gracias, bendiciones infinitas por su vocación, conocimiento y su ayuda. Cambiaron mi vida totalmente. Dios los bendiga"

\section{Discussion}

Se trata de una paciente joven, con trastorno crónico de personalidad ansiosa, altamente exigente consigo misma y con su entorno con un nivel de estrés elevado que afecta su desempeño social. Se encuentra en la evaluación con un trastorno de sueño: Insomnio secundario, depresión como consecuencia del trastorno de sueño crónico, afectación de su rol social y psicosocial, con impacto negativo en su actividad familiar y laboral además con uso y abuso crónico de medicamentos ansiolíticos, antidepresivos e hipnóticos, con efectos citotóxicos y hepatotóxicos, taquifilaxia por uso crónico de medicamentos para el insomnio. Dado lo anterior se realiza un enfoque terapeutico 
diferente realizando un trabajo de regulación por sistemas que finalmente llevara a un equilibrio metabólico, mental y emocional que finalmente controlara el problema de sueño de la paciente. A diferencia de los tratamientos previos recibidos por la paciente este enfoque biorregulador no se concentró en inducir exclusivamente el sueño, sino en modular la respuesta inflamatoria crónica y en buscar un drenaje de la matrix celular. Existe muy poca evidencia de manejo con medicina biorreguladora de pacientes con insomnio sin embargo este caso nos enseña que el trastorno de sueño es una consecuencia del bloqueo metabólico y cómo con una intervención multinivel con medicamentos alopáticos se logra equilibrar la esfera mental, fisica, emocional y metabólica.

\section{Conclusion}

Los trastornos de sueño especialmente el insomnio es muy frecuente en todos los grupos poblacionales. Tiene una realción estrecha con los trastornos de personalidad en especial la ansiedad y depresión que tienen un detonante claro como es el estrés. Cada vez mas los pacientes buscan opciones terapéuticas diferentes de la medicina tradicional en especial por los efectos indeseables como son somnolencia y letargia residual, pérdida del autocontrol y en los casos mas graves sindromes de abstinencia. El inducir directamente el sueño con intervenciones farmacológicas, sin una preparación adecuada del organismo ha llevado a fracasos terapéuticos a través del tiempo. El uso de medicina biorreguladora permite de manera integral modular diferentes sistemas involucrados en la genesis de los trastornos emocionales que finalmente se manifiestan con trastornos de sueño, con la ventaja de ser terapias con mínimos efectos secundarios a pesar de su uso por períodos prolongados. Para este caso pudimos identificar como como en un período de 5 meses, con una intervención integral, la paciente mejora de manera importante y lo pudimos medir en el cuestionario de calidad de vida que previo a la intervención los resultados muestran como los porcentajes estaban por debajo del $50 \%$ y 5 meses después mejoraron con niveles superiores al $85 \%$. Parte importante del éxito de estas terapias está en la comunicación con el paciente, la confianza que se le brinde y la paciencia en el logro de los resultados.

\section{Acknowledgements}

Agradezco a todo mi equipo de trabajo porque me brindaron todo el apoyo para poder tener los resultados que tuvimos con esta paciente, en especial al Dr. Lobelo que con su experiencia, conocimiento y trabajo conjunto logramos sacar adelante este difícil caso de insomnio crónico.

\section{$\underline{\text { References }}$}

1.Medrano-Martínez P Alteraciones cognitivas y emocionales en el insomnio crónico. MJ.Rev Neurol. 16 de febrero de 2016; 62 (4): 170-8.

2. Mallorca. Torrens Prevalencia de insomnio y caracteristicas de pacientes con insomnio en un area de salud de Mallorca.Aten Primaria. Diciembre de 2019; 51 (10): 617-625. doi: 10.1016 / j.aprim.2018.02.014. Epub 20198 de marzo.

3 Peñuela-Epalza MEPrevalencia de insomnio en adultos de 18 a 60 años y exposición a campos electromagneticos en hogares de Barranquilla, Colombia. Biomedica. Agosto de 2015; Especificación 35: 120-9. 
4 Insomnio. Un problema de salud severo.Sanchez-Cárdenas, Rev Med Inst Mex Seguro Soc.

Noviembrediciembre de 2016; 54 (6): 760-769

\section{Attachments}

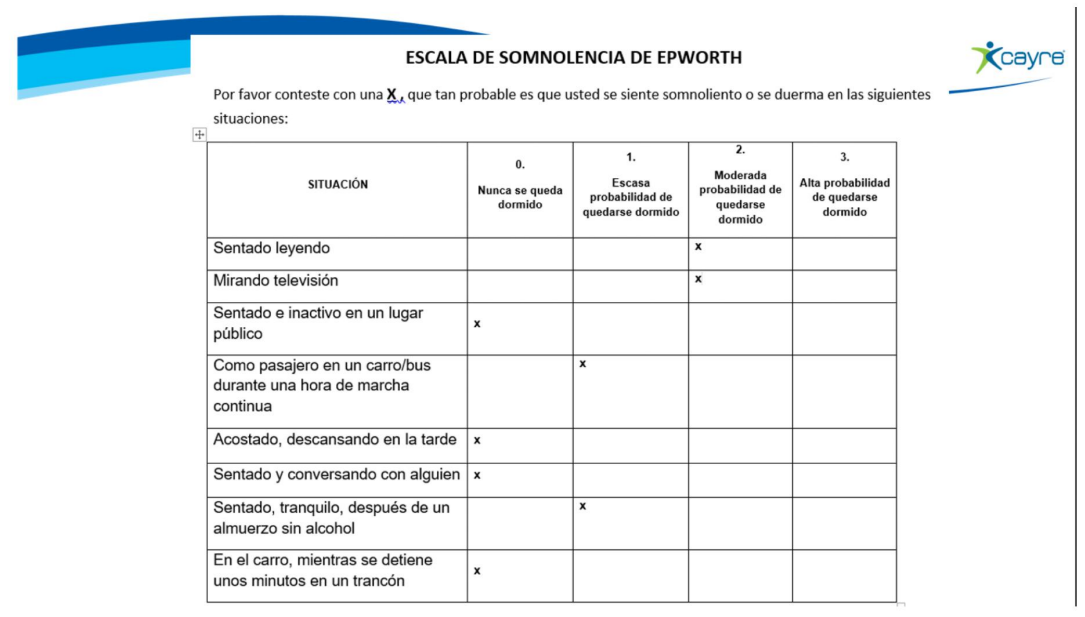

primera escala de Epworth realizada a la paciente en Agosto del 2020 mostrando un resultado de 6/24 


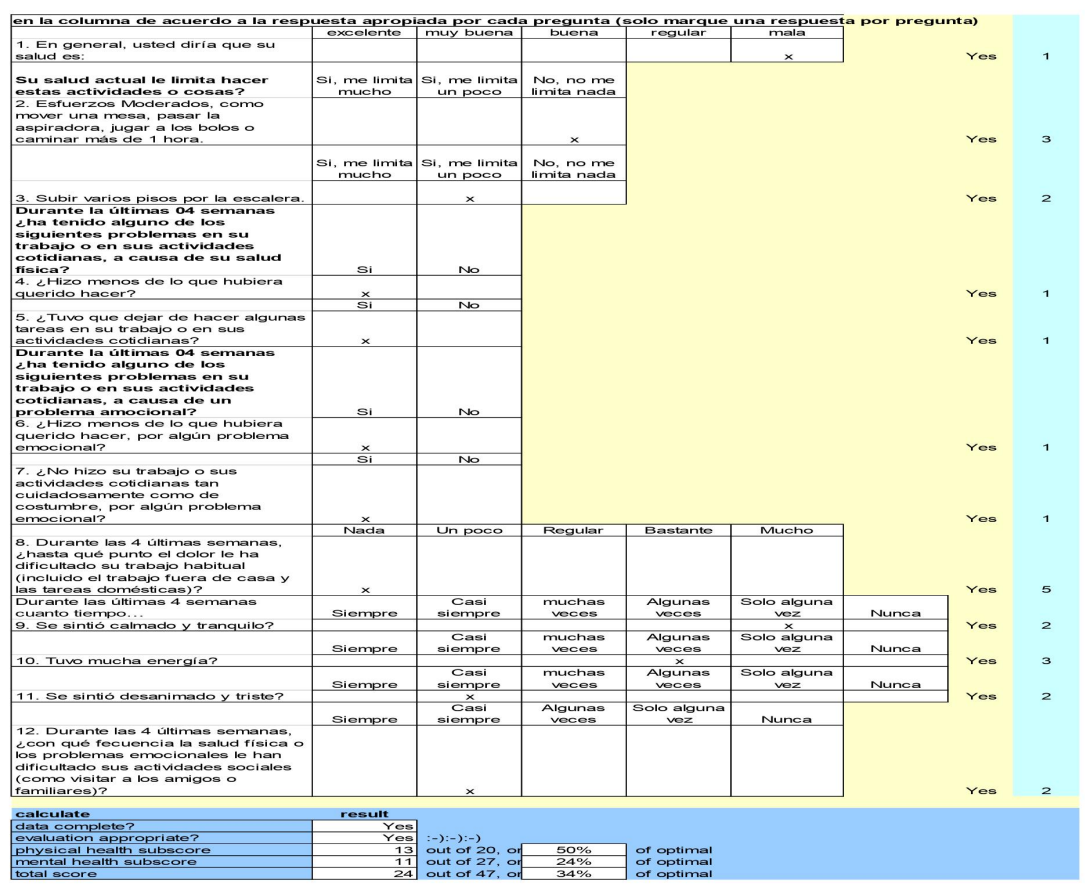

Para la interpretación de este cuestionario de calidad de vida se debe tener encuenta como linea de base 50 puntos y la mejor o peor percepción de calidad de vida se analiza con una desviación estandar de 10 puntos. Esta escala se le practica a la paciente previo a la intervención con medicina biorreguladora. 


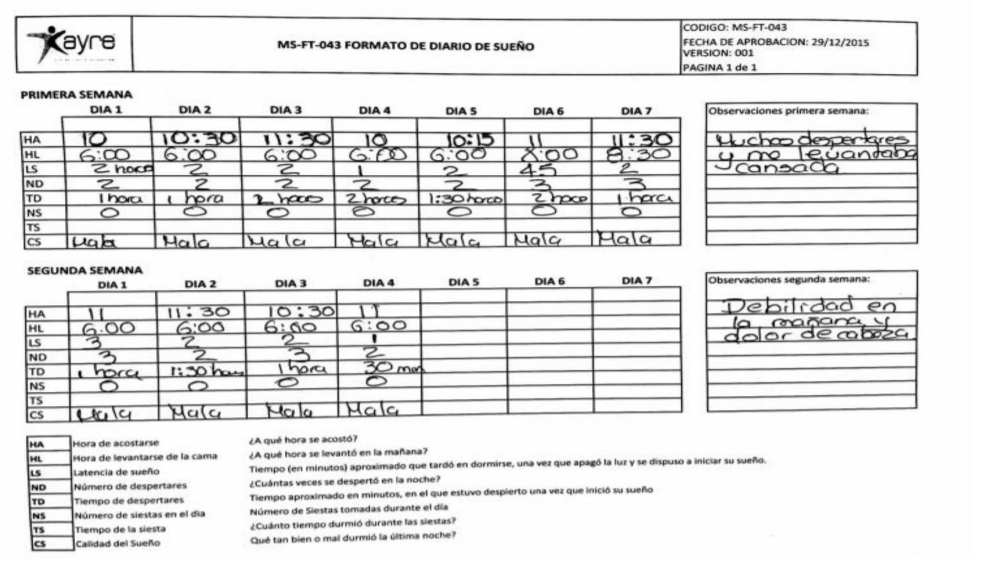

Primer diario de sueño realizado por la paciente en Agosto de 2020 


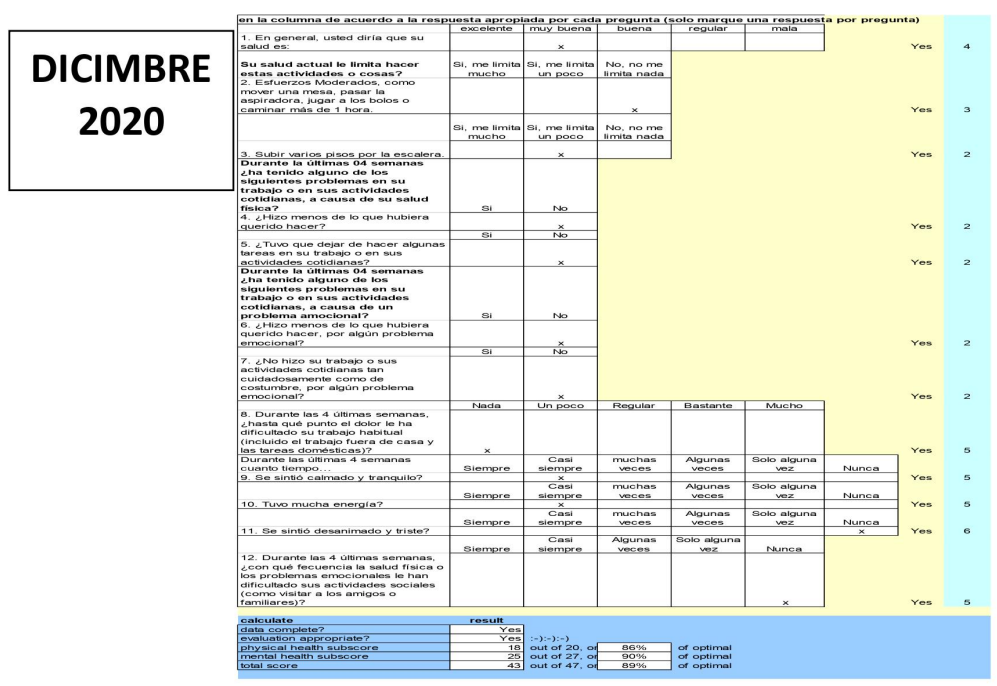

Segundo cuestionario de caliad de vida posterior a intervención con medicina biorreguladora. Diciembre 2020. 


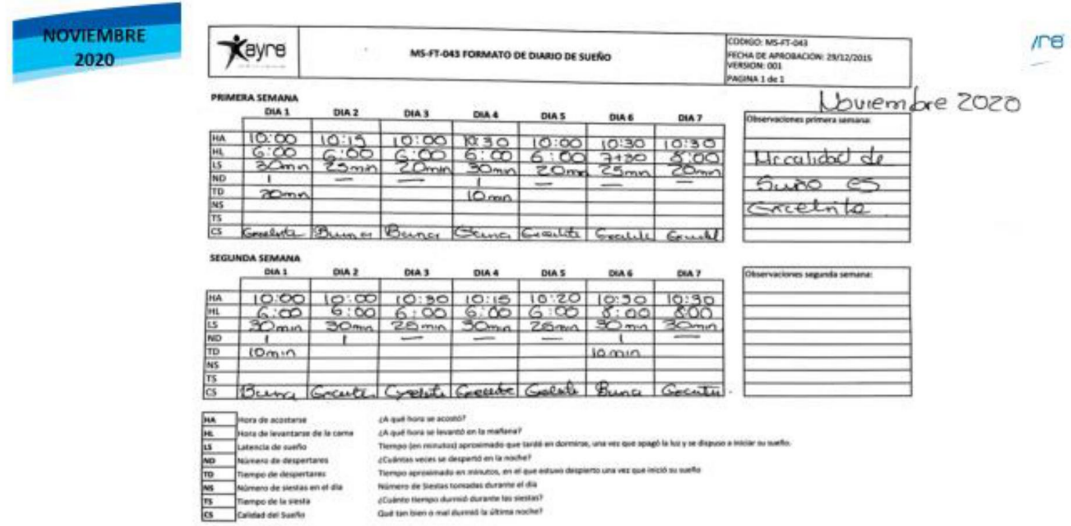

Diario de sueño posterior a intervención con medicina biorreguladora, Noviembre 2020 